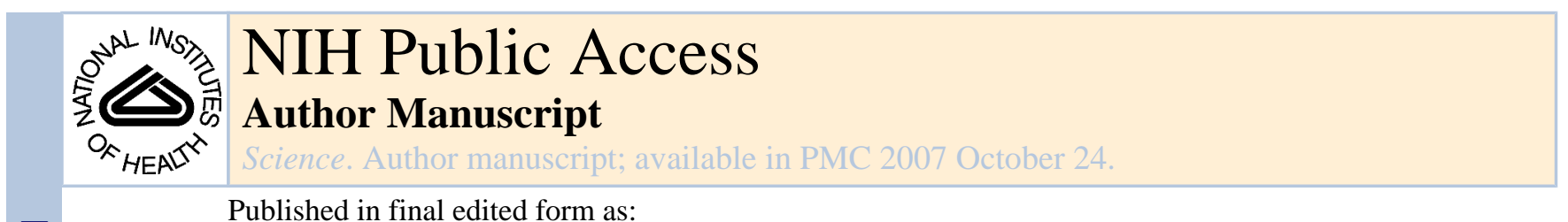

Published in final edited form as:

Science. 2007 January 19; 315(5810): 336-338.

\title{
Is More Neurogenesis Always Better?
}

\author{
Helen E. Scharfman and Rene Hen \\ H. E. Scharfman is in the Departments of Pharmacology and Neurology, Columbia University, and \\ Center for Neural Recovery and Rehabilitation Research, Helen Hayes Hospital, West Haverstraw, \\ NY 10993-1195, USA. R. Hen is in the Departments of Pharmacology and Psychiatry and the \\ Center for Neurobiology and Behavior, Columbia University, New York, NY 10032, USA. E-mail: \\ scharfmanh@helenhayeshosp.org
}

For decades, it was believed that the adult mammalian brain could not generate new neurons, but during the 1990s, that concept changed. Evidence of the birth of new neurons in adult mammals, including humans, raised expectations for improved treatment for patients with central nervous system injury or illness. But this enthusiasm has been tempered since then, as more recent studies indicate that excess adult neurogenesis can be as detrimental as a deficit. In some cases, the clinical relevance of increasing neurogenesis may need to be reconsidered.

Neurogenesis in the normal adult mammalian brain is primarily limited to three areas: the subventricular zone, hippocampal dentate gyrus, and olfactory bulb (1). The identification that this is true in humans, at least in the hippocampus (2), together with the findings that neurogenesis can be increased in laboratory animals by learning, exercise, and antidepressants and decreased by stress and aging (1), reinforced the expectation that neurogenesis might be clinically beneficial. Moreover, additional sites in the adult brain-the cortex and hypothalamus - demonstrate ongoing neurogenesis $(3,4)$, although this remains controversial (5). However, we now know that neurogenesis in the adult brain occurs at a very low rate after maturity, and many of the new neurons do not survive for long (6). Thus, new neurons born in the adult brain may support plasticity on an acute time scale because of their increased excitability (7) but have limited long-term restorative ability. Such transient existence of new neurons should not necessarily dampen therapeutic potential. Survival of new neurons increases with benign interventions such as learning and enriching the environment (1). Dormant stem cells may also exist throughout the brain (8). These cells could potentially be stimulated to mature in pathological situations or after pharmacological interventions. Indeed, a possible reason for the beneficial effects of rehabilitation or psychotherapy may be that treatment increases survival of new neurons.

But an increase in neurogenesis may not always result in improved function. Recent studies show surprising limitations in the ways new neurons in the adult brain can improve function. For example, dentate gyrus neurogenesis influences some hippocampal-dependent behaviors in laboratory animals, but not others. Specifically, there are positive effects on trace and contextual fear conditioning, but not on spatial learning (9-11). Animals without new neurons also perform better in certain working memory paradigms (12). Specifically, mice that are devoid of neurogenesis due to irradiation or genetic ablation display improved memory in a radial maze, but only when repetitive information is presented. Therefore, manipulations that increase neurogenesis may have positive effects on some behaviors but negative effects on others. In addition, improved function may not always be caused by increased neurogenesis. For example, some of the behavioral effects of enriched environment and antidepressants are independent of their influence on hippocampal neurogenesis (13). So despite increasing

The clinical relevance of increasing neurogenesis in the adult mammalian brain is being questioned as increasing the number of new neurons has positive effects on some brain functions but not others. 
experimental support for an influence of neurogenesis on specific behaviors, it is not yet clear how these effects may translate into clinical benefits.

Neurogenesis under pathological conditions also indicates limits to the utility of new neurons in improving brain function. A common theme is that neurogenesis increases after injury to the central nervous system (14). This could be considered restorative, and findings such as the migration of new neurons to the site of damage, at least in animal models of stroke $(15,16)$, support this view. Because pathological conditions also increase the production of factors that promote neurogenesis (brain-derived neuro-trophic factor, vascular endothelial growth factor, insulin-like growth factor, fibroblast growth factor 2, and neuropeptide $\mathrm{Y}$ ), there may be a rapid response of the brain to damage that reflects a recapitulation of developmental programs.

However, the acute increase in neurogenesis and associated growth-related changes are often transient, limiting their influence. Indeed, after an acute increase in neurogenesis, there may be a protracted decline in the rate of neurogenesis (see the figure), and this could contribute to what is often an intractable clinical condition.

Increasing neurogenesis may not always be beneficial in the context of pathology. New neurons may not develop, migrate, or integrate correctly, as in animal models of temporal lobe epilepsy. In such models, severe prolonged seizures (status epilepticus) are followed not only by robust increases in numbers of new granule neurons in the dentate gyrus, but also by inappropriate migration, differentiation, and integration of many of these new neurons (see the figure) (17). This may contribute to persistent seizures in animals, and a similar process may occur in some patients with intractable temporal lobe epilepsy (18).

Some commonly prescribed drugs have robust effects on neurogenesis. These include antidepressants and mood stabilizers (see the Table). Indeed, some of these treatments may ameliorate symptoms because of their effects on neurogenesis, as suggested by studies in animal models $(19,20)$. Specifically, the behavioral effects of selective serotonin reup-take inhibitors and tricyclic antidepressants were blocked in two rodent models of anxiety/ depression by radiological and genetic ablation of neurogenesis in the dentate gyrus (19). How do changes in hippocampal function, presumably caused by neurogenesis, affect mood or anxiety? Although the answer to this question is not clear, ablation of the ventral hippocampus can alter mood, presumably because of its connectivity with limbic structures such as the amygdala, the prefrontal cortex, and the nucleus accumbens (21).

Although neurogenesis occurs throughout life, its clinical potential remains unclear in some cazses. While there is some evidence that strategies to increase neurogenesis may lead to the development of new therapeutics such as antidepressants, decreasing neurogenesis may be beneficial in other cases, such as epilepsy.

\section{References and Notes}

1. Kempermann, G. Adult Neurogenesis: Stem Cells and Neuronal Development in the Adult Brain. Oxford Univ. Press; New York: 2006.

2. Eriksson PS, et al. Nat Med 1998;4:1313. [PubMed: 9809557]

3. Kokoeva MV, Yin H, Flier JS. Science 2005;310:679. [PubMed: 16254185]

4. Dayer AG, Cleaver KM, Abouantoun T, Cameron HA. J Cell Biol 2005;168:415. [PubMed: 15684031]

5. Rakic P. Nat Rev Neurosci 2002;3:65. [PubMed: 11823806]

6. Dayer AG, Ford AA, Cleaver KM, Yassaee M, Cameron HA. J Comp Neurol 2003;460:563. [PubMed: 12717714]

7. Doetsch F, Hen R. Curr Opin Neurobiol 2005;15:121. [PubMed: 15721754]

8. Zhao M, et al. Proc Natl Acad Sci USA 2003;100:7925. [PubMed: 12792021]

9. Saxe MD, et al. Proc Natl Acad Sci USA 2006;103:17501. [PubMed: 17088541] 
10. Shors TJ, Townsend DA, Zhao M, Kozorovitskiy Y, Gould E. Hippocampus 2002;12:578. [PubMed: 12440573]

11. Winocur G, Wojtowicz JM, Sekeres M, Snyder JS, Wang S. Hippocampus 2006;16:296. [PubMed: 16411241]

12. Saxe, M., et al. Society for Neuroscience 2006. Atlanta: Online, Program No. 266.6, 14 to 18 October 2006

13. Meshi D, et al. Nat Neurosci 2006;9:729. [PubMed: 16648847]

14. Kuhn HG, Palmer TD, Fuchs E. Eur Arch Psychiatry Clin Neurosci 2001;251:152. [PubMed: 11697579]

15. Arvidsson A, Collin T, Kirik D, Kokaia Z, Lindvall O. Nat Med 2002;8:963. [PubMed: 12161747]

16. Parent JM, Vexler ZS, Gong C, Derugin N, Ferriero DM. Ann Neurol 2002;52:802. [PubMed: 12447935]

17. Scharfman HE. Adv Med Exp Biol 2004;548:192.

18. Parent JM, Elliott RC, Pleasure SJ, Barbaro NM, Lowenstein DH. Ann Neurol 2006;59:81. [PubMed: 16261566]

19. Santarelli L, et al. Science 2003;301:805. [PubMed: 12907793]

20. Malberg JE, Eisch AJ, Nestler EJ, Duman RS. J Neurosci 2000;20:9104. [PubMed: 11124987]

21. Bannerman DM, et al. Neurosci Biobehav Rev 2004;28:273. [PubMed: 15225971]

22. Longo FM, Yang T, Xie Y, Massa SM. Curr Alzheimer Res 2006;3:5. [PubMed: 16472196]

23. Jin K, Xie L, Mao XO, Greenberg DA. Brain Res 2006;1085:183. [PubMed: 16580645]

24. Keilhoff G, Bernstein HG, Becker A, Grecksch G, Wolf G. Biol Psychiatry 2004;56:317. [PubMed: 15336513]

25. Supported by the National Institute of Neurological Disorders and Stroke, the National Institute of Mental Health, and the National Alliance for Research on Schizophrenia and Depression. 


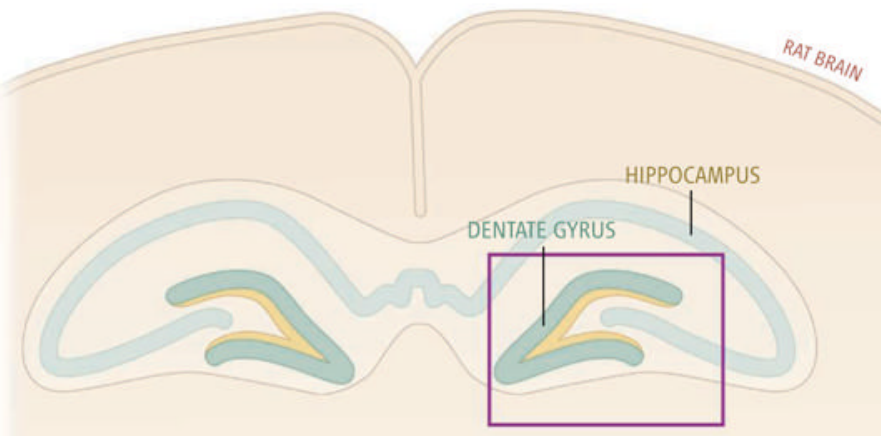

Normal brain neurogenesis

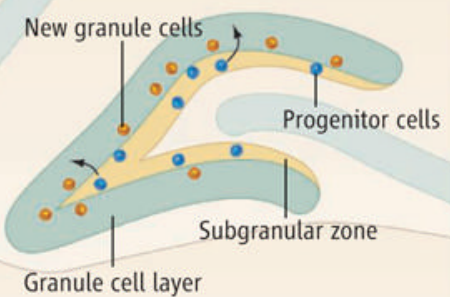

Granule cell layer
Increased neurogenesis

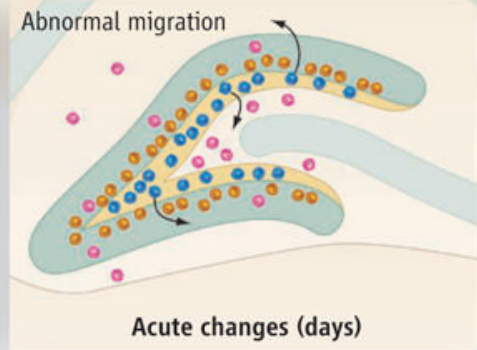

Decreased neurogenesis

Abnormal connections

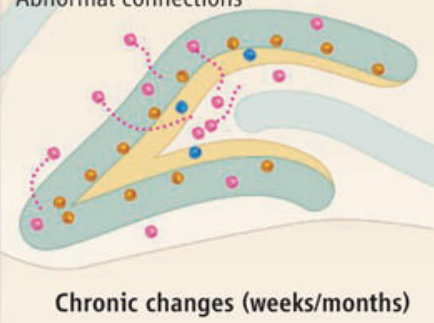

1. Adult hippocampal neurogenesis

Acute and chronic changes in rat brain neurogenesis after severe seizures parallel changes observed in temporal lobe epilepsy. (Left) Most progenitor cells typically become granule cells that migrate to the granule cell layer. (Center) Seizures rapidly and transiently increase the rate of neurogenesis and expression of growth factors that influence neurogenesis. (Right) Ectopic migration of new neurons may result in abnormal neuronal connections. Neurogenesis in laboratory animals and humans may decline at later times, but some of the neurons that were born in the acute period persist. Reduced neurogenesis and growth factor levels, together with abnormal new circuitry, may contribute to the chronic condition. 
Drugs that increase hippocampal neurogenesis

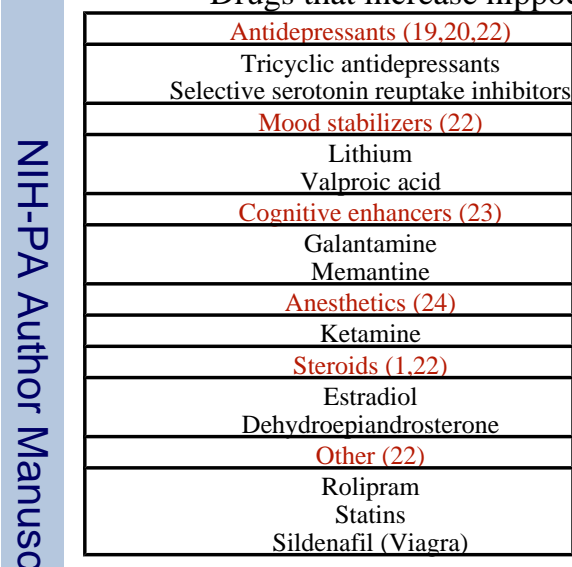

